\title{
Commentary \\ Recently published papers: Renal support in acute kidney injury - is low dose the new high dose?
}

\author{
Yadullah Syed ${ }^{1}$, James AP Tomlinson ${ }^{1}$ and Lui G Forni ${ }^{2}$
}

1Worthing General Hospital, Lyndhurst Road, Worthing, West Sussex BN11 2DH, UK

${ }^{2}$ Brighton and Sussex Medical Schools, University of Sussex, Brighton, East Sussex BN1 9PX, UK

Corresponding author: Lui G Forni, Lui.Forni@wash.nhs.uk

Published: 11 December 2009

This article is online at http://ccforum.com/content/13/6/1014

(c) 2009 BioMed Central Ltd
Critical Care 2009, 13:1014 (doi:10.1186/cc8180)

One of the most studied causes of AKI is contrast-induced nephropathy $(\mathrm{CIN})$. This is due, in part, to the fact that it is easily quantified, but it does also contribute to the burden of $\mathrm{AKI}$ and given the significance of relatively small rises in creatinine, it cannot be ignored. Two recent articles have once again examined potential protective strategies in CIN. Vasheghani-Farahani and colleagues [4] investigated the added benefit of using sodium bicarbonate in addition to $0.9 \%$ saline when compared to $0.9 \%$ saline alone in patients with chronic kidney disease undergoing coronary angiography. The study included 265 patients, with CIN defined by the usual criteria (creatinine rise $>0.5 \mathrm{mg} / \mathrm{dL}$ at 2 and 5 days post-contrast). Little difference was observed between the treatment and control arms (13\% versus $8.6 \%$ ), although the study was somewhat underpowered. A further paper by Majumdar and colleagues [5] attempted to test the protective effect of mannitol and furosemide by creating a forceddiuresis while maintaining euvolaemia with $0.45 \%$ saline in a similar patient group. The results are far from encouraging. The incidence of CIN was greater than expected and significantly worse in the forced diuresis group (50\% versus $28 \%$ ). These studies join the cohort of papers that show little or no clinically relevant benefit in protecting against CIN. In order to reduce this complication, we should focus our energies on those measures we know that do work. Strategies to avoid intravascular depletion or omitting diuretics, nephrotoxins and renal haemodynamic agents are simple but often incompletely instituted. Getting these aspects right could arguably produce more convincing evidence of benefit for our patients than the persistent quest to find the CIN 'magic pill'. In the meantime we are left waiting for the definitive trial on the definitive treatment: whatever that may prove to be.

Despite our best efforts, critically ill patients do, on occasion, require renal support in whatever guise whereas the mainstay

$\mathrm{AKI}=$ acute kidney injury; $\mathrm{CIN}=$ contrast-induced nephropathy; $\mathrm{RRT}=$ renal replacement therapy. 
of chronic renal support remains outpatient haemodialysis. In this setting the dose is commonly quantified in terms of urea kinetics, with urea acting as a surrogate for the clearance of other low-molecular-weight solutes, and there is much evidence that higher dosing regimes are translated into improved outcomes. As a consequence, nephrologists and intensivists alike have embraced the idea that 'high-dose' replacement therapy in the ICU setting must be a good thing. Indeed, initial study seemed to support this [6]. Unfortunately, multi-centre studies do not seem to support this and a recent study published in the New England Journal of Medicine does not lend any further evidence in support of this practice [7]. The Randomized Evaluation of Normal versus Augmented Level Replacement Therapy Study (RENAL) randomly assigned 1,508 ICU patients who required renal support, of which 1,464 were assessed, to receive continuous venovenous hemodiafiltration at one of two 'doses'. Patients were either allotted to a total effluent flow rate of $25 \mathrm{ml} / \mathrm{kg}$ body weight per hour or $40 \mathrm{ml} / \mathrm{kg}$ body weight per hour with treatment continued until recovery of kidney function or discharge from intensive care. The primary outcome being death from any cause at 90 days, secondary outcomes were the usual suspects of need for mechanical ventilation, death within 28 days, death in ICU and cessation of renal replacement therapy (RRT). There was no difference in primary outcome, with $44.7 \%$ of patients dying in the first 90 days after randomization. Encouragingly, $94.4 \%$ of patients who did survive to 90 days no longer required dialysis with similar rates of renal recovery in both treatment groups. Unsurprisingly, the higher dose group demonstrated lower values for the conventional markers of renal function, an increase in the number of filters employed and, more worryingly, an increase in hypophosphataemia. Perhaps one of the most remarkable statistics was the similarity in fluid balance between the two groups, with mean daily balances of just $20 \mathrm{ml}$ being achieved. This is a unique study in that all patients received only continuous therapies and the study therapy was discontinued in dialysis-dependent patients when they left ICU, in contrast to the Acute Renal Failure Trial Network Study [8]. However, no improvement in outcomes with more intensive RRT was demonstrated. So, does this mean that the dose delivered does not matter? We would argue that it does, it is just that the threshold of that dose is yet to be ascertained and perhaps we have been a little enthusiastic regarding the dose prescription; therefore, one wonders whether 'low dose is the new high dose' (JA Kellum, personal communication). What is clear is that in common with most studies the dose prescribed is rarely achieved and surely this must be the aim in order to optimize clinical outcomes, although $88 \%$ of patients did achieve the lower dose regime. Increasing the intensity of therapy beyond the 'new high dose' may not confer additional clinical benefit.

Finally, a study on cessation of therapy. A further subgroup analysis of the BEST kidney data (Beginning and Ending Supportive Therapy for the Kidney) has examined the clinical parameters associated with successful cessation of continuous RRT [9]. This post hoc analysis examined 529 patients. Of these, 313 patients were removed successfully from RRT and did not require any RRT for at least 7 days; these were classified as the 'success' group. The remaining 216 patients were classified as the 'repeat-RRT' group. Those in the 'success' group had a lower hospital mortality (28.5\% versus $42.7 \%, P<0.0001$ ) but also had a lower burden of chronic kidney disease and, perhaps unsurprisingly, lower creatinine and urea concentrations as well as increased urine output. Patients on haemodialysis also seemed to fare less well. Conventional markers also demonstrated improvements with lower creatinine and urea concentrations observed as well as a higher urine output at the time of RRT cessation. Multivariate logistic regression identified urine output in the 24 hours prior to stopping RRT and absolute creatinine concentration as significant predictors for successful cessation. Interestingly, the predictive ability of urine output was negatively affected by the use of diuretics. Perhaps the most practical aspect of this study is that patients who produce more than $400 \mathrm{ml} /$ day of urine without diuretics or $>2,300 \mathrm{ml} /$ day with diuretics have a greater than $80 \%$ chance of successful discontinuation of continuous RRT. Although further prospective studies are needed to test this observation, it may be the first evidence available that can guide our treatment.

\section{Competing interests}

The authors declare that they have no competing interests.

\section{References}

1. Thakar CV, Christianson A, Freyberg R, Almenoff P, Render ML: Incidence and outcomes of acute kidney injury in intensive care units: A Veterans Administration study. Crit Care Med 2009, 37:2552-2558.

2. Lassnigg A, Schmidlin D, Mouhieddine M, Bachmann LM, Druml W, Bauer P, Hiesmayr M: Minimal changes of serum creatinine predict prognosis in patients after cardiothoracic surgery: a prospective cohort study. J Am Soc Nephrol 2004, 15:15971605.

3. Chertow GM, Burdick E, Honour M, Bonventre JV, Bates DW: Acute kidney injury, mortality, length of stay, and costs in hospitalized patients. J Am Soc Nephrol 2005, 16:3365-3370.

4. Vasheghani-Farahni A, Sadigh G, Kassaian SE, Khatami SMR, Fotouhi A, Razavi SAH, Mansournia MA, Yamini-Sharif A, Amirzadegan A, Salarifar M, Sadeghian S, Davoodi G, Borumand MA, Esfehani FA, Darabian S: Sodium bicarbonate plus isotonic saline versus saline for prevention of contrast-induced nephropathy in patients undergoing coronary angiography: a randomized controlled trial. Am J Kidney Dis 2009, 54:610618.

5. Majumdar SR, Kjellstrand CM, Tymchak WJ, Hervas-Malo M, Taylor DA, Teo KK: Forced euvolaemic diuresis with mannitol and furosemide for prevention of contrast-induced nephropathy in patients with CKD undergoing coronary angiography: a randomized controlled trial. Am J Kidney Dis 2009, 54:602-609.

6. Ronco C, Bellomo R, Homel P, Brendolan A, Dan M, Piccinni P, La Greca G: Effects of different doses in continuous venovenous haemofiltration on outcomes of acute renal failure: a prospective randomized trial. Lancet 2000, 355:26-30.

7. The RENAL Study Investigators: Intensity of continuous renalreplacement therapy in critically ill patients. $N$ Engl J Med 2009, 361:1627-1638.

8. The VA/NIH Acute Renal Failure Trial Network: Intensity of renal support in critically ill patients with acute kidney injury. $N$ Engl J Med 2009, 359:7-20. 
9. Uchino S, Bellomo R, Morimatsu H, Morgera S, Schetz M, Tan I, Boumann C, Macedo E, Gibney N, Tolwani A, Oudemas-van Straten, Ronco C, Kellum JA: Discontinuation of continuous renal replacement therapy; a post hoc analysis of a prospective multicentre observational study. Crit Care Med 2009, 37: 2576-2582. 